\title{
Zur Kenntnis der Naphthalaldehydsäure
} von

\author{
phil. stud. Josef Zink.
}

Aus dem chemischen Laboratorium der k. k. deutschen Universität in Prag.

(Vorgelegt in der Sitzung am 23. Mai 1901.)

Vor einigen Jahren wurden von Prof. Goldschmiedt in Gemeinschaft mit L. Egger ${ }^{1}$ die Ester der Opian- und Phtalaldehydsäure in absolut alkoholișcher Lösung mit Cyankalium in Reaction gebracht. Dabei wurden nicht, wie man erwarten sollte, Benzoinderivate, sondern im ersten Falle Tetramethoxyldiphthalyl, im zweiten Diphthalyl erhalten. Goldschmied hat aus diesem Verhalten den Schluss gezogen, dass die Ester in diesem Falle als Pseudoester reagieren nach der Formel:

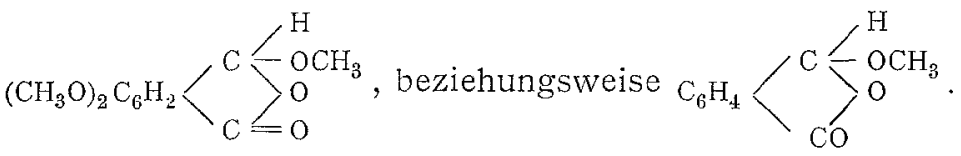

Auch aus dem normalen Ester der Opiansäure war er imstande, ${ }^{2}$ durch Behandeln mit Cyankalium dasselbe Condensationsproduct zu erhalten.

Durch die Arbeiten von Graebe und Gfeller ${ }^{3}$ wurde die Naphthalaldehydsäure leicht zugänglich. Da nun die Periderivate des Naphthalins bis zu einem gewissen Grade große Ähnlichkeit in ihrem Verhalten mit den Orthoderivaten des Benzols zeigen, war es von Interesse, Condensationsversuche

1. Monatshefte für Chemie, XII, 49 .

2 Ebenda, XIII, 263.

3 Liebig's Ann. der Chemie und Pharm., 276, 13. 
der Naphthalaldehydsäure unter dem Einflusse von Cyankalium vorzunehmen. Über Aufforderung des Herrn Prof. Gold$\mathrm{schmiedt} \mathrm{habe} \mathrm{ich} \mathrm{nun} \mathrm{diesbezügliche} \mathrm{Versuche} \mathrm{unternommen.}$

Die Darstellung der Säure selbst geschah nach Graebe und Gfeller ${ }^{1}$ aus Acenaphthenchinon mittels der Kalischmelze. Dabei wurde wahrgenommen, dass stets ein Theil, und zwar umsomehr, je länger erhitzt wurde, in Naphthalsäure übergieng (Schmelzpunkt des Anhydrids $265^{\circ}$ ). Die Naphthalaldehydsäure wurde von der beigemengten Naphthalsäure durch Kochen mit Alkohol getrennt; die Naphthalsäure geht in ihr Anhydrid über und kann als solches wegen seiner Schwerlöslichkeit in Alkohol leicht abgetrennt werden.

Die Naphthalaldehydsäure wurde mehrmals aus verdünntem Alkohol umkrystallisiert und so an reiner (unter Beobachtung der von Graebe und Gfeller angegebenen Vorsichtsmaßregeln) bei $167^{\circ}$ schmelzenden Säure $90 \%$ des angewendeten Ace. naphthenchinons, entsprechend $82 \%$ der theoretischen Ausbeute gewonnen. Daneben wurden selbst bei vorsichtigem Schmelzen 10 bis $12 \%$ Naphthalsäureanhydrid erhalten. Auch beim Kochen der Naphthalaldehydsäure in stark alkalischer Lösung konnte der Übergang in Naphthalsäure constatiert werden, ein Umstand, der an das Verhalten der Phthalaldehydsäure erinnert, welche, wie Hamburge ${ }^{2}$ festgestellt hat, beim Kochen mit Alkali Phthalsäure liefert, sowie an jenes der Opiansäure, welche nach Mathiessen und Forster ${ }^{3}$ bei gleicher Behandlung in Hemipinsäure übergeht. Während aber in diesen Fällen neben Phthalsäure, analog der Bildung von Benzoesäure und Benzylalkohol aus Benzaldehyd, unter dem Einflusse von Alkali, als correspondierender Alkohol das Phthalid, neben Hemipinsäure das Mekonin gebildet wird, gelang es bei der Naphthalaldehydsäure nicht, das Entstehen einer Oxysäure, beziehungsweise eines Lactons nachzuweisen.

Ester der Naphthalaldebyds äure. Bisher waren keine Ester der Naphthalaldehydsäure bekannt. Da nun zu den

1 Liebig's Ann. der Chemie und Pharm., 276, 13.

2 Monatshefte für Chemie, XIX, 427.

3 Ebenda, XII, 49. 
Condensationsversuchen der Naphthalaldehydsäure mit Cyankalium ein Ester der Säure erforderlich war, so gieng ich daran, den Methylester darzustellen. Durch bloßes Kochen der Säure mit absolutem Methylalkohol fand zum Unterschiede von der Opian- und Phthalaldehydsäure keine Esterbildung statt. Es wurde nun die absolut methylalkoholische Lösung der Säure nach dem zuerst von $W$ öhle $\mathrm{r}^{1}$ bei Opiansäure eingeschlagenen Verfahren mit Schwefeldioxyd bei gewöhnlicher Temperatur gesättigt und nach zwölfstündigem Stehen das überschüssige Schwefeldioxyd durch Kochen verjagt. Beim Erkalten der eingeengten Lösung schieden sich anscheinend rhombische Krystalle $a b$, die nach mehrmaligem Umkrystallisieren rein weiß sind und bei $105^{\circ}$ schmelzen. Die Esterificierungsmethode mit trockenem Chlorwasserstoff, die Wegscheider ${ }^{2}$ bei der Opiansäure ein schlechtes Resultat ergab, bewährte sich bei der Naphthalaldehydsäure sehr gut.

$5 \mathrm{~g}$ Säure wurden in $30 \mathrm{~g}$ absolutem Methylalkohol gelöst, und in die Lösung wurde anfangs unter Eiskühlung, später bei gewöhnlicher Temperatur trockenes Salzsäuregas bis zur Sättigung eingeleitet. Am nächsten Tage wurde die alkoholische Lösung in eine gesättigtè Lösung von Natriumbicarbonat gegossen, wobei sich der Ester abschied. Er schmilzt bei $105^{\circ}$ und ist mit dem mit Hilfe von Schwefeldioxyd gewonnenen identisch.

In heißem Wasser ist er ziemlich, in Alkohol, Äther und Benzol leicht löslich. Die Ausbeute ist in beiden Fällen so gut wie quantitativ. Bei der Methoxylbestimmung gaben:

$0.2506 \mathrm{~g}$ Ester $0.2610 \mathrm{~g}$ Jodsilber.

In 100 Theilen:

$$
\mathrm{CH}_{3} \mathrm{O} \ldots \ldots \underbrace{\text { Gefunden }}_{13 \cdot 76} \quad \frac{\mathrm{C}_{13} \mathrm{H}_{10} \mathrm{O}_{3}}{14 \cdot 44}
$$

Bei der Verbrennung gaben: $0.1920 \mathrm{~g}$ Ester $0.5105 \mathrm{~g}$ Kohlendioxyd und $0.0845 \mathrm{~g}$ Wasser.

1 Liebig's Ann., 50, 5.

2 Monatshefte für Chemie, XIII, 711. 
In 100 Theilen:

$\begin{array}{ccc}\mathrm{C} \ldots \ldots \ldots & \begin{array}{r}72 \cdot 51 \\ \mathrm{H} \ldots \ldots \ldots\end{array} & 4 \cdot 88\end{array} \quad \frac{\begin{array}{r}72 \cdot 89 \\ \text { Gefunden }\end{array}}{4 \cdot 67}$

Der Ester wird durch einstündiges Kochen mit Wasser nicht verseift; er reagiert mit salzsaurem Hydroxylamin bei gewöhnlicher Temperatur in alkoholischer Lösung selbst bei tagelangem Stehen nicht. Bei Oximierungsversuchen in alkalischer Lösung bei Zimmertemperatur wurde das bei $257^{\circ}$ schmelzende, von Graebe und Gfeller ${ }^{1}$ zuerst dargestellte Oximanhydrid ${ }^{2}$ der Naphthalaldehydsäure erhalten, welches außer durch seinen Schmelzpunkt durch seine Schwerlöslichkeit in organischen Lösungsmitteln erkannt wurde. Überdies wurde durch das negative Resultat einer Methoxylbestimmung erwiesen, dass der Körper nicht das Oxim des Esters sei.

Wegscheider hat beim Behandeln des Silbersalzes der Opiansäure mit Halogenalkyl einen Ester von anderen Eigenschaften erhalten als beim Kochen der Säure mit Alkohol. Nach seinen Angaben ${ }^{3}$ wurde nun das Silbersalz der Naphthalaldehydsäure mit Hilfe des nach Pfaundler ${ }^{4}$ gewonnenen Fluorsilbers dargestellt. Dasselbe ${ }^{5}$ ist anfangs weiß, färbt sich bald grau, schließlich braunviolett. Das im Vacuum getrocknete Silbersalz wurde fein zerrieben, mit Methylalkohol angerührt und die Lösung mit Jodmethyi versetzt. Aus der nach 24 stündigem Stehen abfiltrierten Lösung konnte derselbe Ester vom

1 Liebig's Ann. der Chemie und Pharm., 276, 13.

2 In Beilstein's Handbuch ist jene Verbindung aufgenommen, welche durch Einwirkung von 2 Molecülen $\mathrm{NH}_{2} \mathrm{OH} . \mathrm{HCl}$ auf Naphthalaldehydsäure entsteht, und dieser irrthümlich der Schmelzpunkt des obigen nicht registrierten Oxims $\left(257^{\circ}\right)$ zugeschrieben.

3 Monatshefte für Chemie, III, 348.

4 Jahresberichte für 1862, S. 88 .

$50.3730 \mathrm{~g}$ des bei $100^{\circ}$ getrockneten Silbersalzes gaben $0.1320 \mathrm{~g} \mathrm{Ag}$. In 100 Theilen: Berechnet für




Schmelzpunkte $105^{\circ}$ isoliert werden, welcher nach den anderen Methoden gewonnen wurde.

Der beschriebene Ester scheint demnach ein wirklicher Ester ${ }^{1} \mathrm{zu}$ sein. Auf die weitere Untersuchung dieserVerhältnisse einzugehen, liegt mir ferne, da ich ja den Ester nur zum Zwecke des Versuches einer bestimmten Reaction dargestellt habe, die in analogen Fällen mit dem normalen Ester ebenso gut geht wie mit dem Pseudoester.

\section{Verhalten der Naphthalaldehydsäure gegen Kaliumcyanid.}

Bei einem Versuche wurden $3 g$ Ester mit $3 g$ bei $110^{\circ}$ getrocknetem Cyankalium in $50 \mathrm{~g}$ absolutem Äthylalkohol $2 \frac{1}{2}$ Stunden gekocht. Sodann wurde vom Rückstande abfiltriert, letzterer in Wasser gelöst und die Lösung mit Salzsäure angesäuert, wobei sich zunächst gelblich gefärbte Nadeln abschieden, die nach mehrmaligem Umkrystallisieren aus Alkohol die gelbe Farbe verloren und sich als Naphthalsäure erwiesen (Schmelzpunkt des Anhydrids $265^{\circ}$ ).

Dasselbe Resultat lieferte ein zweiter Versuch, bei welchem nur $3 / 4$ Stunden erhitzt wurde. Auch aus der vom ungelösten Kaliumcyanid und naphthalsauren Kalium abfiltrierten Lösung konnte nur Naphthalsäure isoliert werden.

Während also die Opiansäure nach Goldschmiedt und Egger.2 unter dem Einflusse von Cyankalium Tetramethoxyldiphthalyl, die Phthalaldehydsäure Diphthalyl liefert, wirkt Cyankalium auf die Naphthalaldehydsäure wie freies Alkali ein.

1 Diese Ansicht hat inzwischen ihre Bestätigung gefunden, da H. Me y e r während des Druckes dieser Abhandlung im hiesigen Laboratorium aus dem unter Anwendung von Thionylchlorid bereiteten Chlorid der Naphtaldehydsäure den Methylester dieser Säure dargestellt und mit meinen Präparaten identisch befunder hat. Meyer beobachtete den Schmelzpunkt 103 bis $104^{\circ}$.

2. Monatshefte für Chemie, 12,49 . 